\title{
Bernstein-Doetsch type results for $(k, h)$-convex functions
}

Attila Házy 


\title{
BERNSTEIN-DOETSCH TYPE RESULTS FOR $(k, h)$-CONVEX FUNCTIONS
}

\author{
ATTILA HÁZY
}

Received 5 September, 2012

\begin{abstract}
In this paper we define the so-called $(k, h)$-convex function which is a natural generalization of the usual convexity, the $s$-convexity in the first and second sense, the $h$-convexity, the Godunova-Levin functions and the $P$-functions.

Some regularity and Bernstein-Doetsch type results are investigated for $(k, h)$-convex functions.
\end{abstract}

2000 Mathematics Subject Classification: 26A51; 26B25; 39B62

Keywords: convexity, h-convexity, s-convexity, Bernstein-Doetsch theorem, regularity properties of generalized convex functions

\section{INTRODUCTION}

In the paper of B. Micherda and T. Rajba [22] were introduced the notions of $k$-convex set and the $(k, h)$-convex functions in the following way:

Let $k:(0,1) \rightarrow \mathbb{R}$ be a given function. Then a subset $D$ of a real linear space $X$ will be called $k$-convex if $k(t) x+k(1-t) y \in D$ for all $x, y \in D$ and $t \in(0,1)$.

Let $k, h:(0,1) \rightarrow \mathbb{R}$ be two given functions and suppose that $D \subset X$ is a $k$-convex set. Then a function $f: D \rightarrow \mathbb{R}$ is $(k, h)$-convex if, for all $x, y \in D$ and $t \in(0,1)$,

$$
f(k(t) x+k(1-t) y) \leq h(t) f(x)+h(1-t) f(y) .
$$

They prove some properties of these sets and functions and gave some example too. Now we introduce notion of $(k, h)$-convexity with respect to $T$.

Let $X$ be a real or complex topological vector space, $T$ be a nonempty set, such that the following property fulfill:

$$
t \in T \text { if and only if } 1-t \in T .
$$

This research has been supported:

1. by the Hungarian Scientific Research Fund (OTKA) Grant NK-81402;

2. as part of the TAMOP-4.2.1.B-10/2/KONV-2010-0001 project with support by the European Union, co-financed by the European Social Fund, and

3. by the János Bolyai Research Scholarship of the Hungarian Academy of Sciences. 
For example $T=\{1 / 2\}, T=\left\{t_{0}, 1-t_{0}\right\}$, or $T$ is an interval (or an arbitrary subset of $\mathbb{R}$ ) which symmetric with respect to $1 / 2$. Furthermore, $T$ may be a subset of $\mathbb{C}$, but it can be a subset of $\mathbb{R}^{n}$ or $\mathbb{C}^{n}$ too.

Furthermore, let $D \subset X$ be a nonempty open, $k$-convex set (that is, $k(t) x+k(1-$ $t) y \in D$ whenever $x ; y \in D$ and $t \in T)$, and $k, h: T \rightarrow \mathbb{R}$ be given functions. We say the function $f: D \rightarrow \mathbb{R}$ is $(k, h)$-convex function, if

$$
f(k(t) x+k(1-t) y) \leq h(t) f(x)+h(1-t) f(y),
$$

for all $x ; y \in D$ and $t \in T$. To avoid the trivialities and the unimportant cases, we suppose that there exists an element $t_{0} \in T$ such that

$$
k\left(t_{0}\right) k\left(1-t_{0}\right) h\left(t_{0}\right) h\left(1-t_{0}\right) \neq 0 .
$$

In the present paper we investigate these $(k, h)$-convex functions. This notion of convexity is a natural generalization of the usual convexity, the $s$-convexity in first and second sense, the $h$-convexity, the Godunova-Levin functions and the $P$ functions.

In the special cases when $T=\{1 / 2\}, T=\left\{t_{0}, 1-t_{0}\right\}$ or $T=\mathbb{Q} \cap[0,1]$, the corresponding convex functions are said to be Jensen- $(k, h)$-convex, $t_{0}-(k, h)$-convex and rationally- $(k, h)$-convex.

The convex functions $f$

$$
f(t x+(1-t) y) \leq t f(x)+(1-t) f(y) .
$$

are obviously $(k, h)$-convex functions with $k(t)=t, h(t)=t$.

Let $h:[0,1] \rightarrow \mathbb{R}$ be a given function. In the case, when $k(t)=t$ we get the so called $h$-convex functions, which was introduced by Varošanec [33] and was generalized by Házy [12]. We say that $f: D \rightarrow \mathbb{R}$ is an $h$-convex function if, for all $x, y \in D$ and $t \in[0,1]$, we have

$$
f(t x+(1-t) y) \leq h(t) f(x)+h(1-t) f(y) .
$$

The Godunova-Levin functions were investigated in [9]. We say that $f: I \rightarrow \mathbb{R}$ (where $I$ is a real interval) is a Godunova-Levin function, if $f$ is nonnegative and for all $x, y \in I$ and $t \in(0,1)$ we have

$$
f(t x+(1-t) y) \leq \frac{f(x)}{t}+\frac{f(y)}{1-t} .
$$

Some properties of this type of functions are given in [23] by Dragomir, Pečaric̀ and Persson, [7] by Mitrinovic̀ and Pečaric̀, [24] by Mitrinovic̀, Pečaric̀ and Fink. Among others, it is proved that nonnegative monotone and nonnegative convex functions belong to this class of functions. The Godunova-Levin functions are $(k, h)$-convex functions, with $k(t)=t, h(t)=1 / t$.

The concept of $s$-convexity in the first sense was introduced in [26] by Orlicz. A real valued function $f: D \rightarrow \mathbb{R}$ is called Orlicz s-convex or s-convex in the first 
sense, if

$$
f\left(t^{s} x+(1-t)^{s} y\right) \leq t f(x)+(1-t) f(y)
$$

for every $x, y \in D, t \in] 0,1]$, where $s \in[1, \infty[$ is a fixed number. The Orlicz $s$-convex functions are $(k, h)$-convex functions, with $k(t)=t^{s}, h(t)=t$.

The concept of $s$-convexity in the second sense was introduced in [2] by Breckner. A real valued function $f: D \rightarrow \mathbb{R}$ is called Breckner s-convex or s-convex in the second sense, if

$$
f(t x+(1-t) y) \leq t^{s} f(x)+(1-t)^{s} f(y)
$$

for every $x, y \in D$ and $t \in[0,1]$, where $s \in] 0,1]$ is a fixed number. The Breckner $s$-convex functions are $(k, h)$-convex functions, with $k(t)=t, h(t)=t^{s}$.

In [2,3] (by Breckner) and [4] (by Breckner and Orban) Berstein-Doetsch type results were proved on rationally $s$-convex functions, moreover, for the $s$-Hölder property of $s$-convex functions. In [31] Pycia gave a new proof of the latter statement, when $f$ is defined on a nonempty, convex subset of a finite dimensional vector space. In the paper [15] the authors Hudzik and Maligranda collected some properties of $s$-convex functions defined on the nonnegative half line. In the paper [5] (by Burai, Házy and Juhász) there are some Berstein-Doetsch type results on the so-called $(H, s)$-convex functions.

The $P$-functions were investigated in [7] by Dragomir, Pečaric̀ and Persson. A real valued function $f: D \rightarrow \mathbb{R}$ (where $D$ is a convex, open, nonempty subset of a real (complex) linear space $X$ ) is called $P$-function, if for every $x, y \in D$ and $t \in[0,1]$ we have

$$
f(t x+(1-t) y) \leq f(x)+f(y) .
$$

Some results about the $P$-functions can be found in [30] by Pearce and Rubinov, [32] by Tseng, Yang and Dragomir. The $P$-functions are $(k, h)$-convex functions, with $k(t)=t, h(t)=1$.

In [1] Bernstein and Doetsch proved that if a function $f: D \rightarrow \mathbb{R}$ (where $D$ is a convex, open, nonempty subset of a real (complex) linear space $X$ ) is locally bounded from above at a point of $D$, then the Jensen-convexity of the function yields its local boundedness and continuity as well, which implies the convexity of the function $f$ (see Kuczma [16] for further references). This result has been generalized by several authors. The first such type results are due to Nikodem and $\mathrm{Ng}$ [25] for the approximately Jensen-convex functions (the so-called $\varepsilon$-Jensen-convexity), which was extended by Páles $([28,29])$ to approximately $t$-convex functions. Further generalizations can be found in papers of Házy [10,11], Házy and Páles [13,14], Makó and Páles [17-20], Maksa and Páles [21] and M. E. Özdemir, A. Ekinci and A. O. Akdemir [27]. In the paper [8] by Gilányi, Nikodem and Páles there are some BernsteinDoetsch type results for quasiconvex functions. 


\section{MAIN RESULTS}

In the sequel let $(X,\|\cdot\|)$ is a real (complex) normed space. We recall that a function $f: D \rightarrow \mathbb{R}$ (where $D \subset X$ ) is referred to as locally bounded from above on $D$ if, for each point $p \in D$, there exist $\varrho>0$ and a neighborhood $U(p, \varrho):=\{x \in$ $X:\|x-p\|<\varrho$ \} such that $f$ is bounded from above on $U(p, \varrho)$. We assume that $k, h: T \rightarrow[0, \infty[$.

Proposition 1. Let $t_{0} \in T$ be fixed such that $k\left(t_{0}\right)+k\left(1-t_{0}\right)=1$ and $f: D \rightarrow \mathbb{R}$ be a $t_{0}-(k, h)$-convex function. Then we have

(i) if $h\left(t_{0}\right)+h\left(1-t_{0}\right)>1$ then $f$ is nonnegative.

(ii) if $h\left(t_{0}\right)+h\left(1-t_{0}\right)<1$ then $f$ is nonpositive.

Proof. Let $x$ be an arbitrary element of $D$. Using $(k, h)$-convexity of $f$

$$
\begin{aligned}
f(x) & =f\left(k\left(t_{0}\right) x+k\left(1-t_{0}\right) x\right) \\
& \leq h\left(t_{0}\right) f(x)+h\left(1-t_{0}\right) f(x)=\left(h\left(t_{0}\right)+h\left(1-t_{0}\right)\right) f(x),
\end{aligned}
$$

which implies

$$
0 \leq\left(h\left(t_{0}\right)+h\left(1-t_{0}\right)-1\right) f(x)
$$

If $h\left(t_{0}\right)+h\left(1-t_{0}\right)-1>0$, then we have $f(x) \geq 0$ and if $h\left(t_{0}\right)+h\left(1-t_{0}\right)-1<0$, then we have $f(x) \leq 0$.

We remark, that if $h\left(t_{0}\right)+h\left(1-t_{0}\right)=1$, then, similarly the usual convexity and Jensen-convexity, there is no such type result. An easy consequence of the previous proposition is the following:

Corollary 1. Let $h, k: T \rightarrow \mathbb{R}$ be given functions such that $k(t)+k(1-t)=1$ for all $t \in T$, and let $f: D \rightarrow \mathbb{R}$ be an $T-(k, h)$-convex function. Then

(i) if $h(t)+h(1-t) \geq 1$ for all $t \in T$ and there exists $t_{1} \in T$ such that $h\left(t_{1}\right)+$ $h\left(1-t_{1}\right)>1$, then $f$ is nonnegative.

(ii) if $h(t)+h(1-t) \leq 1$ for all $t \in T$ and there exists $t_{1} \in T$ such that $h\left(t_{1}\right)+$ $h\left(1-t_{1}\right)<1$, then $f$ is nonpositive.

(iii) if there exists $t_{1}, t_{2} \in T$ such that $h\left(t_{1}\right)+h\left(1-t_{1}\right)>1$ and $h\left(t_{2}\right)+h\left(1-t_{2}\right)<$ 1 then $f$ is constant 0 .

The proof of this result is similar to the proof of Proposition 1.

Some immediate consequences of this proposition are expressed in the following two corollaries. The second one shows that every Breckner $s$-convex function is nonnegative.

Corollary 2. Let $f: D \rightarrow \mathbb{R}$ be a $\left(t_{0}, h\right)$-convex function. Then

(i) if $h\left(t_{0}\right)+h\left(1-t_{0}\right)>1$, then $f$ is nonnegative.

(ii) if $h\left(t_{0}\right)+h\left(1-t_{0}\right)<1$, then $f$ is nonpositive.

Corollary 3. Every Breckner $\left(t_{0}, s\right)$-convex function is nonnegative. 
In our next results, we deal with the boundedness property of $(k, h)$-convex functions. We assume that $h(t)$ and $h(1-t)$ are not zero simultaneously. Because, if $h(t)=h(1-t)=0$, then the $(h, k)$-convexity not implies the boundedness of $f$. In this case we get $f(x) \leq 0$ from the inequality (1.1), that is, we get only the upper boundedness of $f$. The proof is analogous to those in [12].

Theorem 1. Let $k, h: T \rightarrow \mathbb{R}$ be given nonnegative functions and let $t_{0} \in T$ be fixed such that $k\left(t_{0}\right) k\left(1-t_{0}\right) \neq 0$ and $k\left(t_{0}\right)+k\left(1-t_{0}\right)=1$. We assume that $h\left(t_{0}\right)$ and $h\left(1-t_{0}\right)$ are not zero simultaneously. Furthermore let $D \subset X$ be an open, nonempty, $k$-convex set, and let $f: D \rightarrow \mathbb{R}$ be a $t_{0^{-}}(k, h)$-convex function. Then

(i) if $h\left(t_{0}\right)+h\left(1-t_{0}\right)<1$ or

(ii) if $h\left(t_{0}\right)+h\left(1-t_{0}\right) \geq 1$ and $f$ is locally bounded from above at a point $p \in D$ then $f$ is locally bounded at every point of $D$.

Proof. Since $k\left(t_{0}\right) k\left(1-t_{0}\right) \neq 0$ therefore we get $k\left(t_{0}\right), k\left(1-t_{0}\right)>0$. Because of the property $h\left(t_{0}\right)$ and $h\left(1-t_{0}\right)$ are not zero simultaneously, therefore, without loss generality, we may assume that $h\left(t_{0}\right)>0$. In the case $(i)$ the local upper boundedness yields from Proposition 1 . So we prove that $\mathrm{f}$ is locally bounded from above on $D$ in the case $(i i)$.

First we prove that $f$ is locally bounded from above on $D$. Define the sequence of sets $D_{n}$ by

$$
D_{0}:=\{p\}, \quad D_{n+1}:=k\left(t_{0}\right) D_{n}+k\left(1-t_{0}\right) D .
$$

Using induction on $n$, we prove that $f$ is locally upper bounded at each point of $D_{n}$. By assumption, $f$ is locally bounded from above at $p \in D_{0}$. Assume that $f$ is locally upper bounded at each point of $D_{n}$. For $x \in D_{n+1}$, there exist $x_{0} \in D_{n}$ and $y_{0} \in D$ such that $x=k\left(t_{0}\right) x_{0}+k\left(1-t_{0}\right) y_{0}$. By the inductive hypothesis, there exist $r>0$ and a constant $M_{0} \geq 0$ with $f\left(x^{\prime}\right) \leq M_{0}$ such that $\left\|x_{0}-x^{\prime}\right\|<r$. Then, by the $t_{0}-(k, h)$-convexity of $f$, for $x^{\prime} \in U_{0}:=U\left(x_{0}, r\right)$ we have

$$
\begin{aligned}
f\left(k\left(t_{0}\right) x^{\prime}+k\left(1-t_{0}\right) y_{0}\right) & \leq h\left(t_{0}\right) f\left(x^{\prime}\right)+h\left(1-t_{0}\right) f\left(y_{0}\right) \\
& \leq h\left(t_{0}\right) M_{0}+h\left(1-t_{0}\right) f\left(y_{0}\right)=: M .
\end{aligned}
$$

Therefore, for

$$
\begin{aligned}
y \in U & :=k\left(t_{0}\right) U_{0}+k\left(1-t_{0}\right) y_{0}=U\left(k\left(t_{0}\right) x_{0}+k\left(1-t_{0}\right) y_{0}, k\left(t_{0}\right) r\right) \\
& =U\left(x, k\left(t_{0}\right) r\right),
\end{aligned}
$$

we get that $f(y) \leq M$. Thus $f$ is locally bounded from above on $D_{n+1}$. On the other hand, we can show that

$$
D=\bigcup_{n=1}^{\infty} D_{n} .
$$


Since the inclusion $\bigcup_{n=1}^{\infty} D_{n} \subseteq D$ is obvious we just check the reverse inclusion. In fact, from the definition of $D_{n}$, it follows by induction that $D_{n}=\left(k\left(t_{0}\right)\right)^{n} p+(1-$ $\left.\left(k\left(t_{0}\right)\right)^{n}\right) D$. For fixed $x \in D$, define the sequence $x_{n}$ by

$$
x_{n}:=\frac{x-\left(k\left(t_{0}\right)\right)^{n} p}{1-\left(k\left(t_{0}\right)\right)^{n}} .
$$

Then $x_{n} \rightarrow x$ if $n \rightarrow \infty$. As $D$ is open, $x_{n} \in D$ for some $n$. Therefore,

$$
x=\left(k\left(t_{0}\right)\right)^{n} p+\left(1-\left(k\left(t_{0}\right)\right)^{n}\right) x_{n} \in\left(k\left(t_{0}\right)\right)^{n} p+\left(1-\left(k\left(t_{0}\right)\right)^{n}\right) D=D_{n},
$$

which the converse inclusion. Thus $f$ is locally bounded from above on $D$.

Now, we prove that $f$ is locally bounded from below. Let $q \in D$ be arbitrary. Since $f$ is locally bounded from above at the point $q$, there exist $\varrho>0$ and $M>0$ such that

$$
\sup _{x \in U(q, \varrho)} f(x) \leq M \text {. }
$$

Let $x \in U\left(q, k\left(1-t_{0}\right) \varrho\right)$ and $y:=\frac{q-k\left(t_{0}\right) x}{k\left(1-t_{0}\right)}$. Then $y$ is in $U(q, \varrho)$. By $t_{0^{-}}(k, h)$ convexity,

which implies

$$
f(q) \leq h\left(t_{0}\right) f(x)+h\left(1-t_{0}\right) f(y)
$$

$$
f(x) \geq \frac{f(q)-h\left(1-t_{0}\right) f(y)}{h\left(t_{0}\right)} \geq \frac{f(q)-h\left(1-t_{0}\right) M}{h\left(t_{0}\right)}=: M^{\prime} .
$$

Therefore $f$ is locally bounded from below at any point of $D$.

The consequence of this theorem is that the local upper boundedness at a point of every convex (or Jensen-convex, or Breckner $s$-convex) function implies its locally boundedness.

Corollary 4. Let $f: D \rightarrow \mathbb{R}$ be a Jensen-convex or $t_{0}$-convex function. If $f$ is locally bounded from above at a point of $D$, then $f$ is locally bounded at every point of $D$.

Corollary 5. Let $f: D \rightarrow \mathbb{R}$ be a Breckner $\left(t_{0}, s\right)$-convex function. If $f$ is locally bounded from above at a point of $D$, then $f$ is locally bounded at every point of $D$.

Corollary 6. Let $f: D \rightarrow \mathbb{R}$ be a $\left(t_{0}, h\right)$-convex function such that $h\left(t_{0}\right)$ and $h\left(1-t_{0}\right)$ are not zero simultaneously. If $f$ is locally bounded from above at a point of $D$, then $f$ is locally bounded at every point of $D$.

It is a well-known fact that if a Jensen-convex function $f$ is locally bounded above at a point of its domain (see $[1,16])$, then it is continuous on the domain.

The nonnegative monotone functions - which are not necessarily continuous - belong to the class of the Godunova-Levin functions (see [7,23,24]), which is a special 
class of $(h, k)$-convex functions. In [5] we gave an example of function which is Jensen-s-convex, bounded and nowhere continuous.

These examples show that the $t_{0}-(k, h)$-convexity of a function and the locally upper boundedness at a point in its domain does not imply the continuity of a $t_{0^{-}}$ $(k, h)$ convex functions.

The next theorem provides a sufficient condition for the local boundedness to imply the continuity.

Theorem 2. Let $T=\left\{t_{n}\right\}_{n \in \mathbb{N}}$ such that the sequence $\left\{t_{n}\right\}_{n \in \mathbb{N}} \subset[0,1]$ tends to 0 , as $n \rightarrow \infty$. Let $k, h: T \rightarrow[0, \infty[$ be given nonnegative continuous functions such that $h\left(t_{n}\right)$ and $h\left(1-t_{n}\right)$ are not simultaneously zero, $k\left(t_{n}\right)+k\left(1-t_{n}\right)=1$ for every $n \in \mathbb{N}$ and the limit conditions

$$
\lim _{t \rightarrow 0} h(t)=0 \quad \text { and } \quad \lim _{t \rightarrow 1} h(t)=1
$$

are valid. Furthermore let $D \subset X$ be an open, nonempty, $k$-convex set. If $f: D \rightarrow \mathbb{R}$ is a $T-(k, h)$-convex function and locally bounded from above at a point of $D$, then $f$ is continuous on $D$.

Proof. Since $h\left(t_{n}\right)$ and $h\left(1-t_{n}\right)$ are not zero simultaneously, therefore, without loss generality, we may assume that $h\left(1-t_{n}\right)>0$.

Since $f$ is locally bounded from above at a point $x_{0} \in D$, there exists a neighborhood $U$ at $x_{0}$ and a constant $K>0$ such that $f(x) \leq K$ for every $x \in U$. Let $\varepsilon$ be an arbitrary nonnegative constant. Then there exists $n_{0} \in \mathbb{N}$ such that if $n \geq n_{0}$, then

$$
h\left(t_{n}\right) K+\left[h\left(1-t_{n}\right)-1\right] f\left(x_{0}\right)<\varepsilon,
$$

whence

$$
\frac{h\left(t_{n}\right)}{h\left(1-t_{n}\right)} K+\left[1-\frac{1}{h\left(1-t_{n}\right)}\right] f\left(x_{0}\right)<\varepsilon .
$$

Let $V$ be a neighborhood of 0 such that $x_{0}+V \subseteq U$, and let $U^{\prime}=x_{0}+k\left(t_{n}\right) V$. We prove that

$$
\left|f(x)-f\left(x_{0}\right)\right|<\varepsilon \quad\left(x \in U^{\prime}\right) .
$$

For $x \in U^{\prime}$ there exist $y, z \in x_{0}+V$ such that

$$
\begin{aligned}
x & =k\left(t_{n}\right) y+k\left(1-t_{n}\right) x_{0}=k\left(t_{n}\right) y+\left(1-k\left(t_{n}\right)\right) x_{0}, \\
x_{0} & =k\left(t_{n}\right) z+k\left(1-t_{n}\right) x=k\left(t_{n}\right) z+\left(1-k\left(t_{n}\right)\right) x .
\end{aligned}
$$

Indeed,

and

$$
y-x_{0}=\frac{1}{k\left(t_{n}\right)}\left(x-x_{0}\right) \in \frac{1}{k\left(t_{n}\right)} k\left(t_{n}\right) V=V,
$$

$$
z-x_{0}=\frac{1-k\left(t_{n}\right)}{k\left(t_{n}\right)}\left(x_{0}-x\right) \in \frac{1-k\left(t_{n}\right)}{k\left(t_{n}\right)} k\left(t_{n}\right) V=\left(1-k\left(t_{n}\right)\right) V \subseteq V
$$


According to $T$ - $(k, h)$-convexity of $f$,

$$
\begin{aligned}
f(x) & \leq h\left(t_{n}\right) f(y)+h\left(1-t_{n}\right) f\left(x_{0}\right) \leq h\left(t_{n}\right) K+h\left(1-t_{n}\right) f\left(x_{0}\right), \\
f\left(x_{0}\right) & \leq h\left(t_{n}\right) f(z)+h\left(1-t_{n}\right) f(x) \leq h\left(t_{n}\right) K+h\left(1-t_{n}\right) f(x) .
\end{aligned}
$$

We get

$$
f(x)-f\left(x_{0}\right) \leq h\left(t_{n}\right) K+\left[h\left(1-t_{n}\right)-1\right] f\left(x_{0}\right)<\varepsilon
$$

and

which implies

$$
f(x) \geq \frac{f\left(x_{0}\right)-h\left(t_{n}\right) K}{h\left(1-t_{n}\right)},
$$

$$
f(x)-f\left(x_{0}\right) \geq\left[\frac{1}{1-h\left(t_{n}\right)}-1\right] f\left(x_{0}\right)-\frac{h\left(t_{n}\right)}{h\left(1-t_{n}\right)} K>-\varepsilon .
$$

The inequalities (2.1) and (2.2) show that $\left|f(x)-f\left(x_{0}\right)\right|<\varepsilon$, that is $f$ is continuous at $x_{0}$, which was to be proved.

The previous limit conditions are not necessary, since in the case of Jensen-convex functions they do not fulfill. However, the result of Bernstein and Doetsch is valid for Jensen-convex functions.

\section{CONVEXITY PROPERTY OF RATIONALLY- $(k, h)$-CONVEX FUNCTIONS}

The following result offers a generalization of the theorem of Bernstein-Doetsch [1], Breckner [2], Burai-Házy-Juhász [5,6] and Házy [12] for rationally- $(k, h)$-convex functions.

Theorem 3. Let $k, h$ be given nonnegative, continuous functions satisfying the limit conditions

$$
\lim _{t \rightarrow 0} h(t)=0 \quad \text { and } \quad \lim _{t \rightarrow 1} h(t)=1 .
$$

and $k(t)+k(1-t)=1$, and assume that $h(t)$ and $h(1-t)$ are not zero simultaneously for all $t \in \mathbb{Q} \cap[0,1]$. Furthermore let $D \subset X$ be an open, nonempty, $k$-convex set. If $f: D \rightarrow \mathbb{R}$ is rationally- $(k, h)$-convex and locally bounded from above at a point of $D$, then $f$ is continuous and $(k, h)$-convex.

Proof. We prove that the function $f$ is $t_{0}-(k, h)$-convex for all $t_{0} \in[0,1]$. Let $t_{0} \in[0,1]$ be arbitrary. Then there exists a sequence $\left\{t_{n}\right\}_{n \in \mathbb{N}}$ such that $t_{n} \in \mathbb{Q} \cap[0,1]$ and $t_{n} \rightarrow t_{0}$ (when $n$ tends to $\infty$ ). Applying rationally- $(k, h)$-convexity of $f$, we get

$$
f\left(k\left(t_{n}\right) x+k\left(1-t_{n}\right) y\right) \leq h\left(t_{n}\right) f(x)+h\left(1-t_{n}\right) f(y) .
$$

The local upper boundedness of $f$ implies the continuity of $f$ (according to Theorem 2). Therefore, taking the limit $n \rightarrow \infty$ in (3.1), we get

$$
f\left(k\left(t_{0}\right) x+k\left(1-t_{0}\right) y\right) \leq h\left(t_{0}\right) f(x)+h\left(1-t_{0}\right) f(y),
$$

which proves the $(k, h)$-convexity of $f$. 
Corollary 7. Let $D \subset X$ be a nonempty, convex, open set and let $h:[0,1] \rightarrow \mathbb{R}$ be a given nonnegative, continuous function satisfying the limit conditions

$$
\lim _{t \rightarrow 0} h(t)=0 \quad \text { and } \quad \lim _{t \rightarrow 1} h(t)=1 .
$$

and assume that $h\left(t_{0}\right)$ and $h\left(1-t_{0}\right)$ are not simultaneously zero for all $t_{0} \in \mathbb{Q} \cap[0,1]$.

If $f: D \rightarrow \mathbb{R}$ is rationally-h-convex and $f$ is locally bounded from above at a point $D$, then $f$ is continuous on $D$ and h-convex.

Corollary 8. Let $D \subset X$ be a nonempty, convex, open set. If $f: D \rightarrow \mathbb{R}$ is rationally-Breckner s-convex and locally bounded from above at a point $D$, then $f$ is continuous on D and Breckner s-convex.

Theorem 4. Let $T=[0,1], k, h: T \rightarrow \mathbb{R}$ be given nonnegative functions such that $k$ is continuous on $T$, and $h(t)+h(1-t)=1$. Let $\gamma(t)=k(t)+k(1-t)$ and $f: \mathbb{R}_{+} \rightarrow \mathbb{R}$ be a $(k, h)$-convex function. Then

(i) $f$ is nondecreasing if $\gamma(T)=[r, 1]($ where $r<1)$.

(ii) $f$ is nonincreasing if $\gamma(T)=[1, r]$ (where $r>1$ ).

(ii i ) $f$ is constant if $\gamma(T)=\left[r_{1}, r_{2}\right]$ (where $r_{1}<1<r_{2}$ ).

Proof. We have, for $x>0$ and $t \in[0,1]$

$$
f(k(t) x+k(1-t) x) \leq h(t) f(x)+h(t) f(x)=f(x) .
$$

Since $\gamma(t)=k(t)+k(1-t)$ and $k$ is continuous on $[0,1]$, therefore $\gamma$ is continuous on $[0,1]$.

In the case $(i)$ we get $\gamma(T)=[r, 1]$, where $r<1$. Let $u \in[r, 1]$ be arbitrary. Then there exists a $t \in[0,1]$ such that $\gamma(t)=u$. This yields that

$$
f(u x) \leq f(x) \quad\left(x \in \mathbb{R}_{+}, u \in[r, 1]\right) .
$$

If now $u \in\left[r^{2}, 1\right]$ then $u^{1 / 2} \in[r, 1]$. Therefore, by the fact that (3.2) holds for all $x \in \mathbb{R}_{+}$, we get

$$
f(u x)=f\left(u^{1 / 2}\left(u^{1 / 2} x\right)\right) \leq f\left(u^{1 / 2} x\right) \leq f(x)
$$

for all $x \in \mathbb{R}_{+}$. By induction we then obtain that

$$
\left.\left.f(u x) \leq f(x) \quad\left(x \in \mathbb{R}_{+}, u \in\right] 0,1\right]\right) .
$$

Therefore, taking $0<x<y$ and applying (3.3), we get

$$
f(x)=f((x / y) y) \leq f(y),
$$

which means that $f$ is nondecreasing on $\mathbb{R}_{+}$.

The proof of the cases (i i) and (ii i) are similar.

The above results do not hold, in general, in the case of convex functions, because a convex function $f: \mathbb{R}_{+} \rightarrow \mathbb{R}$, need not be non-decreasing. But in the case of Orlicz $s$-convex function this is true. 
Corollary 9. Let $0<s<1$. Let $f: \mathbb{R}_{+} \rightarrow \mathbb{R}$ an Orlicz s-convex function. Then $f$ is nondecreasing.

Remark 1. In the paper of Hudzik and Maligranda [15] was given an example showing that the Orlicz $s$-convex function is nondecreasing on $] 0, \infty[$, but not necessarily on $[0, \infty[$. For the readers convenience we recall the example: let $a, b, c \in \mathbb{R}$ and let

$$
f(x)= \begin{cases}a & \text { if } x=0 \\ b x^{s}+c & \text { if } x \neq 0 .\end{cases}
$$

If $b>0$ and $c<a$ then $f$ is non-decreasing on $] 0, \infty[$ but not on $[0, \infty[$.

\section{OPTIMIZATION}

It is a very well known fact that every local minimizer of a convex function is a global one. The same is true for $(k, h)$-convex functions under some assumptions.

Theorem 5. Let $D \subset X$ be a nonempty, open, $k$-convex set, where $k, h:[0,1] \rightarrow \mathbb{R}$ are given nonnegative, continuous functions satisfying the limit conditions

$$
\lim _{t \rightarrow 0+} k(t)=0 \quad \text { and } \quad \lim _{t \rightarrow 1-} k(t)=1 .
$$

and assume that $h(t)+h(1-t)=1$ for all $t \in[0,1]$. Then every local minimizer of $a(k, h)$-convex function $f: D \rightarrow \mathbb{R}$ is a global one.

Furthermore if the function $f$ is strictly $(k, h)$-convex (there is strict inequality in (1.1) whenever $x \neq y$ ), then there is at most one global minimum.

Proof. Let $x_{0} \in D$ be a local minimizer of $f$. Then there exists a positive real number $r$, such that

$$
f\left(x_{0}\right) \leq f(y), \quad y \in U\left(x_{0}, r\right) .
$$

Assume that $x_{0}$ is not a global minimizer. Then there exists $z \in D$, such that $f\left(x_{0}\right)>$ $f(z)$. Using this and the $(k, h)$-convexity of $f$, we have

$$
\begin{aligned}
f\left(k(t) z+k(1-t) x_{0}\right) & \leq h(t) f\left(x_{0}\right)+h(1-t) f(z) \\
& =f\left(x_{0}\right)+h(1-t)\left(f(z)-f\left(x_{0}\right)\right)<f\left(x_{0}\right) .
\end{aligned}
$$

On the other hand, using the limit conditions, $k(t) z+k(1-t) x_{0} \in U\left(x_{0}, r\right)$, if $t$ is sufficiently small, which contradicts the fact that $x_{0}$ is a local minimizer.

If $f$ is a strictly $(k, h)$-convex function, and $x \neq y$ are global minimizers, then

$$
f(k(t) x+k(1-t) y)<h(t) f(x)+h(1-t) f(y)=f(x),
$$

which is a contradiction.

Corollary 10. Every local minimizer of an Orlicz-convex function $f: D \rightarrow \mathbb{R}$ is a global one. If the function $f$ is strictly Orlicz-convex, then there is at most one global minimum.

Corollary 11. Every local minimizer of a convex function $f: D \rightarrow \mathbb{R}$ is a global one. If the function $f$ is strictly convex, then there is at most one global minimum. 


\section{ACKNOWLEDGEMENT}

The author thanks Nutefe Kwami Agbeko for helpful comments.

\section{REFERENCES}

[1] F. Bernstein and G. Doetsch, "On the theory of convex functions. (Zur Theorie der konvexen Funktionen)," Math. Ann., vol. 76, pp. 514-526, 1915.

[2] W. W. Breckner, "Stetigkeitsaussagen für eine Klasse verallgemeinerter konvexer Funktionen in topologischen linearen Räumen,” Publ. Inst. Math., Nouv. Sér., vol. 23(37), pp. 13-20, 1978.

[3] W. W. Breckner, Rational s-convexity. A generalized Jensen-convexity. Cluj-Napoca: Cluj University Press, 2011.

[4] W. W. Breckner and G. Orban, Continuity properties of rationally s-convex mappings with values in an ordered topological linear space. Cluj-Napoca: "Babes-Bolyai" University, Department of Mathematics, 1978.

[5] P. Burai, A. Házy, and T. Juhász, "Bernstein-Doetsch type results for $s$-convex functions," Publ. Math., vol. 75, no. 1-2, pp. 23-31, 2009.

[6] P. Burai, A. Házy, and T. Juhász, "On approximately Breckner $s$-convex functions," Control and Cybernetics, vol. 40, no. 1, pp. 91-99, 2011.

[7] S. S. Dragomir, J. E. Pečarić, and L. E. Persson, "Some inequalities of Hadamard type," Soochow J. Math., vol. 21, no. 3, pp. 335-341, 1995.

[8] A. Gilányi, K. Nikodem, and Z. Páles, "Bernstein-Doetsch type results for quasiconvex functions," Math. Inequal. Appl., vol. 7, no. 2, pp. 160-175, 2004.

[9] E. K. Godunova and V. I. Levin, "Neravenstva dlja funkcii sirokogo klassa, soderzascego vypuklye, monotonnye i nekotorye drugie vidy funkii," Vycislitel. Mat. i. Fiz. Mezvuzov. Sb. Nauc. Trudov, MGPI, Moskva, pp. 138-142, 1985.

[10] A. Házy, "On approximate $t$-convexity," Math. Inequal. Appl., vol. 8, no. 3, pp. 389-402, 2005.

[11] A. Házy, "On the stability of $t$-convex functions," Aequationes Math., vol. 74, no. 3, pp. 210-218, 2007.

[12] A. Házy, "Bernstein-doetsch type results for $h$-convex functions," Math. Inequal. Appl., vol. 14, no. 3, pp. 499-508, 2011.

[13] A. Házy and Z. Páles, "On approximately midconvex functions," Bull. Lond. Math. Soc., vol. 36, no. 3, pp. 339-350, 2004.

[14] A. Házy and Z. Páles, "On approximately $t$-convex functions," Publ. Math., vol. 66, no. 3-4, pp. 489-501, 2005.

[15] H. Hudzik and L. Maligranda, "Some remarks on s-convex functions," Aequationes Math., vol. 48, no. 1, pp. 100-111, 1994.

[16] M. Kuczma, An introduction to the theory of functional equations and inequalities. Cauchy's equation and Jensen's inequality. Warszawa-Kraków-Katowice: Uniwersytet Slaski, 1985.

[17] J. Makó and Z. Páles, "Approximate convexity of Takagi type functions," J. Math. Anal. Appl., vol. 369, no. 2, pp. 545-554, 2010.

[18] J. Makó and Z. Páles, "Strengthening of strong and approximate convexity," Acta Math. Hung., vol. 132, no. 1-2, pp. 78-91, 2011.

[19] J. Makó and Z. Páles, "Implications between approximate convexity properties and approximate Hermite-Hadamard inequalities," Cent. Eur. J. Math., vol. 10, no. 3, pp. 1017-1041, 2012.

[20] J. Makó and Z. Páles, “On $\varphi$-convexity," Publ. Math. Debrecen, vol. 80, no. 1-2, pp. 107-126, 2012.

[21] G. Maksa and Z. Páles, "The equality case in some recent convexity inequalities," Opuscula Math., vol. 31, no. 2, pp. 269-277, 2011. 
[22] B. Micherda and T. Rajba, "On some Hermite-Hadamard-Fejér inequalities for (k,h)-convex functions," Math. Ineq. Appl., vol. 12, no. 4, pp. 931-940, 2012.

[23] D. S. Mitrinović and J. E. Pečarić, "Note on a class of functions of Godunova and Levin," C. R. Math. Acad. Sci., Soc. R. Can., vol. 12, no. 1, pp. 33-36, 1990.

[24] D. S. Mitrinović, J. E. Pečarić, and A. M. Fink, Classical and new inequalities in analysis, ser. Mathematics and Its Applications. East European Series. Dordrecht: Kluwer Academic Publishers, 1993, vol. 61 .

[25] C. T. Ng and K. Nikodem, "On approximately convex functions," Proc. Am. Math. Soc., vol. 118, no. 1, pp. 103-108, 1993.

[26] W. Orlicz, "A note on modular spaces. i," Bull. Acad. Pol. Sci., Sér. Sci. Math. Astron. Phys., vol. 9, pp. 157-162, 1961.

[27] M. E. Özdemir, A. Ekinci, and A. O. Akdemir, "Generalizations of integral inequalities for functions whose second derivatives are convex and m-convex," Miskolc Math. Notes, vol. 13, no. 2, 2012.

[28] Z. Páles, "Bernstein-Doetsch-type results for general functional inequalities," Rocz. Nauk.Dydakt., Pr. Mat., vol. 17, pp. 197-206, 2000.

[29] Z. Páles, "On approximately convex functions," Proc. Am. Math. Soc., vol. 131, no. 1, pp. 243252, 2003.

[30] C. E. M. Pearce and A. M. Rubinov, " $p$-functions, quasi-convex functions, and Hadamard-type inequalities," J. Math. Anal. Appl., vol. 240, no. 1, pp. 92-104, 1999.

[31] M. Pycia, "A direct proof of the $s$-Hölder continuity of Breckner $s$-convex functions," Aequationes Math., vol. 61, no. 1-2, pp. 128-130, 2001

[32] K. L. Tseng, G. S. Yang, and S. S. Dragomir, "On quasi-convex functions and hadamard's inequality," RGMIA Res. Rep. Coll., vol. 6, no. 3, p. Article 1, 2003.

[33] S. Varošanec, "On h-convexity," J. Math. Anal. Appl., vol. 326, no. 1, pp. 303-311, 2007.

Author's address

Attila Házy

Department of Applied Mathematics, University of Miskolc, H-3515 Miskolc-Egyetemváros, Hungary

E-mail address: matha@uni-miskolc.hu 